Pesq. Vet. Bras. 37(8):835-839, agosto 2017 DOI: $10.1590 / \mathrm{S} 0100-736 \mathrm{X} 2017000800009$

\title{
Avaliação clínica de cães com doença do disco intervertebral (Hansen tipo I) submetidos à descompressão cirúrgica: $110 \operatorname{casos}^{1}$
}

\author{
Rafael O. Chaves ${ }^{2 *}$, Dakir N. Polidoro ${ }^{2}$, João P.S. Feranti², Andrei K. Fabretti ${ }^{3}$, Bruna \\ Copat $^{4}$, Lucas A. Gomes ${ }^{3}$ e Alexandre Mazzanti ${ }^{5}$
}

\begin{abstract}
Chaves R.O., Polidoro D.N., Feranti J.P.S., Fabretti A.K., Copat B., Gomes L.A. \& Mazzanti A. 2017. [Clinical evaluation of dogs with intervertebral disc disease (Hansen type I) submitted to surgical decompression: 110 cases.] Avaliação clínica de cães com doença do disco intervertebral (Hansen tipo I) submetidos à descompressão cirúrgica: 110 casos. Pesquisa Veterinária Brasileira 37(8):835-839. Departamento de Clínica de Pequenos Animais, Universidade Federal de Santa Maria, Av. Roraima 1000, Santa Maria, RS 97105-900, Brazil. E-mail: rafaelochaves@hotmail.com

The aim of this study was to identify dogs with thoracolumbar intervertebral disc disease (Hansen type I) submitted for surgical treatment on a routine service in neurology in a university veterinary hospital in 2006-2014, and to get information about age, sex, breed, site of extrusion, interpretation of myelography, evaluation of the degree of neurological dysfunction, functional recovery of these dogs, and to verify the recurrence of clinical signs of disease. The most common breed was Dachshund (69\%), followed by mixed breed (14.5\%). The most affected sites were between T12-T13 (31.9\%) and L1-L2 (19.1\%). Of the 110 dogs, $74(67.3 \%)$ improved clinical signs after surgery, $54(49.1 \%)$ satisfactory and $20(18.2 \%)$, in part satisfactory. From these dogs, six (8.1\%) dogs were in grade II, 19 $(25.7 \%)$ in grade III, $35(47.3 \%)$ in grade IV, and $14(18.9 \%)$ in grade V. It can be concluded that satisfactory surgical treatment promotes functional recovery in most dogs with thoracolumbar disk extrusion. The prognosis for functional recovery after surgical treatment is better the lower the degree of neurological dysfunction and the recurrence percentage is lower in dogs subjected to this type of therapy.
\end{abstract}

INDEX TERMS: Neurology, intervertebral disc disease, Hansen type I, surgical decompression, spinal cord, extrusion, nociception, canine, pet animals.

RESUMO.- 0 objetivo deste estudo foi identificar cães com doença do disco intervertebral toracolombar (Hansen tipo I) submetidos ao tratamento cirúrgico em um serviço de rotina em neurologia de um hospital veterinário universitário no período de 2006 a 2014, e obter informações a respeito

\footnotetext{
${ }^{1}$ Recebido em 29 de junho de 2015.

Aceito para publicação em 4 de setembro de 2016.

${ }^{2}$ Programa de Pós-Graduação em Medicina Veterinária, área de concentração em Cirurgia, Centro de Ciências Rurais (CCR), Universidade Federal de Santa Maria (UFSM), Camobi, Santa Maria, RS 97105-900, Brasil.

${ }^{3}$ Departamento de Clínicas Veterinárias, Universidade Estadual de Londrina (UEL), Rodovia Celso Garcia Cid Km 380, Londrina, PR 86057-970, Brasil.

${ }^{4}$ Programa de Residência do Curso de Medicina Veterinária, Universidade Luterana do Brasil (ULBra), Bairro São José, Canoas, RS 92425-900, Brasil.

${ }^{5}$ Departamento de Clínica de Pequenos Animais, CCR-UFSM, Santa Maria, RS. *Autor para correspondência: rafaelochaves@hotmail.com
}

da idade, do sexo, da raça, local da extrusão, interpretação da mielografia, avaliação dos graus de disfunção neurológica, recuperação funcional desses cães e observar a ocorrência de recidiva dos sinais clínicos da doença. A raça mais frequente foi Dachshund (69\%), seguida dos cães sem raça definida $(14,5 \%)$. Os locais mais afetados foram entre T12-T13 (31,9\%) e L1-L2 (19,1\%). Dos 110 cães, 74 (67,3\%) tiveram melhora dos sinais clínicos após o procedimento cirúrgico, sendo $54(49,1 \%)$ considerados satisfatórios e $20(18,2 \%)$, parcialmente satisfatórios. Destes cães, seis $(8,1 \%)$ cães estavam em grau II, 19 (25,7\%) em grau III, 35 $(47,3 \%)$ em grau IV e $14(18,9 \%)$ em grau V. Pode-se concluir que o tratamento cirúrgico promove recuperação funcional satisfatória na maioria dos cães com extrusão de disco toracolombar. 0 prognóstico para recuperação funcional após o tratamento cirúrgico é tanto melhor quanto menor for o grau de disfunção neurológica e o percentual de recidiva é baixo em animais submetidos a este tipo de terapia. 
TERMOS DE INDEXAÇÃO: Neurologia, medula espinhal, doença do disco intervertebral, Hansen tipo I, descompressão cirúrgica, extrusão, nocicepção, caninos, animais de companhia.

\section{INTRODUÇÃO}

A doença do disco intervertebral (DDIV) é uma das causas mais comuns de alterações neurológicas em cães (Brisson 2010, Fingeroth \& Thomas 2015), sendo provocada pela degeneração do disco intervertebral. Pode ocorrer extrusão (Hansen tipo I) ou protrusão (Hansen tipo II) do disco, as quais podem causar compressão da medula ou das raízes nervosas e até concussão medular (Sharp \& Wheeler 2005, Fingeroth \& Thomas 2015). É observada com frequência em raças condrodistróficas, com idade entre três e sete anos (Aikawa et al. 2012, Kranenburg et al. 2013) e, embora trabalhos relataram uma predisposição em machos (Itoh et al. 2008, Aikawa et al. 2012), a maioria dos estudos indica que a incidência da DDIV é semelhante entre os gêneros (Brisson 2010).

Os sinais clínicos são variáveis e dependem da localização da lesão na medula espinhal, do volume de material no interior do canal vertebral e da velocidade com que esse material é ejetado, variando desde hiperestesia espinhal até paraplegia com perda da dor profunda. 0 tratamento de cães com DDIV toracolombar pode ser clínico ou cirúrgico, dependendo do grau de disfunção neurológica (Brisson 2010, Fingeroth \& Thomas 2015).

As indicações para o tratamento cirúrgico da DDIV são a falta de resposta ao tratamento clínico, sinais clínicos recidivantes ou progressivos, paraparesia não-ambulatória, paraplegia com preservação (grau IV) ou ausência da dor profunda (grau V) com duração inferior a 48 horas (Sharp \& Wheeler 2005, Brisson 2010, Fingeroth \& Thomas 2015). Existem várias técnicas cirúrgicas para a descompressão da medula espinhal na DDIV, sendo a hemilaminectomia, pediculectomia, laminectomia dorsal e corpectomia lateral as mais utilizadas (Sharp \& Wheeler 2005, Brisson 2010, Kerwin et al. 2102). Os objetivos do tratamento cirúrgico na DDIV são: a descompressão da medula espinhal, a remoção do material do interior do canal vertebral, a redução do edema, o alívio da dor e a prevenção de futuras extrusões (Sharp \& Wheeler 2005, Fingeroth \& Thomas 2015).

Vários trabalhos vêm mostrando resultados satisfatórios do tratamento cirúrgico em cães com DDIV toracolombar com diferentes graus de disfunção neurológica (Kazakos et al. 2005, Aikawa et al. 2012, Ingram et al. 2013, Kranenburg et al. 2013). No Brasil, entretanto, poucos estudos são encontrados em cães com essa enfermidade e submetidos ao tratamento cirúrgico (Arias et al. 2007, Festugatto et al. 2008, Santos et al. 2012). Diante disso, o objetivo deste trabalho é identificar cães com doença do disco intervertebral toracolombar submetidos ao tratamento cirúrgico em um serviço de rotina em neurologia de um hospital veterinário universitário e obter informações a respeito da idade, do sexo, da raça, local da extrusão, interpretar a mielografia, avaliar os graus de disfunção neurológica, recuperação funcional desses cães e observar a ocorrência de recidiva dos sinais clínicos da doença.

\section{MATERIAL E MÉTODOS}

Foram revisados os arquivos do Serviço de Neurologia (SN) do Hospital Veterinário Universitário (HVU) da Universidade Federal de Santa Maria (UFSM) no período entre janeiro de 2006 e agosto de 2014. Foram incluídos nesse estudo, apenas cães que apresentavam dados completos, que incluíam histórico clínico e resultados dos exames físico geral e neurológico. A avaliação neurológica dos cães incluiu: observações gerais (nível de consciência e comportamento), análise da postura e marcha, avaliação de nervos cranianos, avaliação das reações posturais, reflexos segmentares espinhais, palpação epaxial (hiperestesia) e presença da dor profunda (nocicepção). Esse último teste foi feito apenas nos animais paraplégicos com auxílio de uma pinça hemostática aplicada em uma das falanges, sendo considerado ausente de dor profunda aqueles que não reagiram ao estímulo doloroso com vocalização, olhar para o local ou inquietude (Sharp \& Wheeler 2005 , Fingeroth \& Thomas 2015). Com base nos resultados desses exames neurológicos e de acordo com o grau de deficiências neurológicas, os casos foram classificados em: Grau I = somente dor à palpação epaxial; Grau II - ataxia proprioceptiva; Grau III = paraparesia; Grau IV = paraplegia com presença de dor profunda; e Grau V = paraplegia com ausência de dor profunda (Sharp \& Wheeler 2005).

0 diagnóstico presuntivo, em todos os animais, foi obtido por meio do histórico, achados clínicos, neurológicos e dos exames complementares, como radiografia simples e contrastada (mielografia). Esse último exame foi feito para confirmar a suspeita clínica de compressão extramedular, em que se incluia a DDIV toracolombar. 0 diagnóstico definitivo foi feito pela visualização, durante o procedimento cirúrgico, do conteúdo do material do núcleo pulposo extrudido no interior do canal vertebral.

Todos os cães incluídos neste estudo foram submetidos ao tratamento cirúrgico. A técnica realizada foi a hemilaminectomia dorsolateral seguida da fenestração do disco intervertebral afetado (Sharp \& Wheeler 2005, Fingeroth \& Thomas 2015). A avaliação da recuperação funcional consistiu de duas formas, (1) exame clínico e neurológico realizado pelo médico veterinário do SN durante o retorno previamente agendado após procedimento cirúrgico e (2) os proprietários dos cães foram contatados por telefonemas para a obtenção das seguintes informações: se houve recuperação funcional satisfatória, insatisfatória ou se houve recidiva dos sinais clínicos. Foram considerados casos recidivantes, aqueles em que os animais foram reavaliados e apresentaram diagnóstico presuntivo de DDIV toracolombar ou cervical e/ou reoperados, confirmando a extrusão de disco intervertebral toracolombar.

A recuperação funcional foi classificada como satisfatória, quando os animais retornaram a caminhar sem quedas e auxílio; parcialmente satisfatória, quando retornaram a caminhar sem auxílio, porém com ataxia e quedas esporádicas; e insatisfatória, quando os sinais neurológicos iniciais permaneceram inalterados. A relação entre grau de disfunção neurológica e recuperação funcional foi analisada por meio do teste qui-quadrado. 0 valor de $\mathrm{P}<0,05$ foi considerado estatisticamente significativo.

\section{RESULTADOS E DISCUSSÃO}

Foram localizadas e revisadas 408 fichas clínicas de cães com DDIV toracolombar atendidos no SN do HVU-UFSM entre janeiro de 2006 e agosto de 2014. Destes, 110 estavam de acordo com os critérios pré-estabelecidos e 298 foram excluídos devido à falta de informações nos registros médicos do HVU-UFSM e/ou permaneceram com o diagnóstico presuntivo e realizaram tratamento clínico. A dis- 
tribuição etária variou de três a 16 anos, com uma média de seis anos de idade, sendo $54,5 \%$ fêmeas (60 de 110cães) e $45,5 \%$ machos (50 de 110 cães). Quanto às raças, Dachshund foi a mais acometida (69\%), seguida de cães sem raça definida $(14,5 \%)$, Poodle $(3,6 \%)$, Cocker spaniel inglês $(2,7 \%)$, Lhasa apso $(1,8 \%)$, Buldog inglês $(1,8 \%)$ e Buldog francês $(1,8 \%)$. 0 que indica que cães de raças condrodistróficas são mais prodispostos que cães não condrodistróficos (Smolders et al. 2013, Kranenburg et al. 2013). Isso ocorre devido a um processo degenerativo que se inicia nos primeiros meses de vida, que resulta no aparecimento precoce de calcificação do disco, tornando-se mais propensos a herniação aguda do material mineralizado do núcleo pulposo para dentro do canal vertebral (Smolders et al. 2013). Resultados semelhantes foram encontrados nos estudos de Aikawa et al. (2012) e Kranenburg et al. (2013) em relação a raça, porém quanto ao sexo, diferente do presente estudo, machos foram mais prevalentes em cães com DDIV toracolombar.

A DDIV é mais comumente associada à compressão extramedular nos espaços intervertebrais entre T11 e L3 (Itoh et al. 2008, Brisson 2010, Aikawa et al. 2012), o que pode ser justificado pela variação anatômica, visto que, do primeiro ao décimo disco intervertebral da região torácica, o ligamento longitudinal dorsal é reforçado pelos ligamentos intercapitais, sendo rara a ocorrência de protrusão ou extrusão de disco nesse segmento (Brisson 2010). No presente estudo, 4 (3,6\%) extrusões ocorreram entre T10-T11, 15 (13,6\%) entre T11-T12, $35(31,9 \%)$ entre T12-T13, 19 (17,3\%) entre T13-L1, 21 (19,1\%) entre L1-L2, $11(10 \%)$ entre L2-L3, um $(0,9 \%)$ entre L3-L4, três $(2,7 \%)$ entre L4-L5 e um (0,9\%) entre L5-L6 (Fig.1). Dos 110 cães avaliados, apenas um cão apresentou compressão medular envolvendo mais de um disco (T12-T13 e L1-L2); resultados semelhantes a estes foram identificados por Aikawa et al. (2012), que citaram a ocorrência única de compressão medular em $93,6 \%$ e $98,2 \%$ dos cães avaliados, respectivamente.

Quanto ao grau de disfunção neurológica, 7 cães $(6,4 \%)$ foram classificados em grau II, 22 cães (20\%) em grau III, 46 cães $(41,8 \%)$ em grau IV e 35 cães $(31,8 \%)$ em grau V (Quadro 1). Este número de cães classificados entre graus III e V tratados cirurgicamente está de acordo com Sharp \& Wheeler (2005) e Fingeroth \& Thomas (2015), os quais re- comendaram a terapia cirúrgica para cães com sinais compatíveis com esses graus de disfunção neurológica. Os cães em grau $\mathrm{V}$ foram submetidos à cirurgia descompressiva antes de completarem 72 horas de ausência de dor profunda, visto que, a recuperação funcional nesses pacientes submetidos à cirurgia descompressiva é melhor quando realizada dentro deste período (Brisson 2010, Fingeroth \& Thomas 2015). Entretanto, Santos et al. (2011), relataram que cães com ausência de dor profunda durante um período superior a 48 horas e não submetidos ao tratamento cirúrgico também podem apresentar recuperação funcional satisfatória, sendo necessários, no mínimo, 30 dias do início dos sinais clínicos para estabelecer um prognóstico quanto ao retorno dos movimentos voluntários. Além disso, cabe ressaltar, que não existem dados referentes à recuperação funcional de cães com DDIV em grau V de disfunção neurológica, e submetidos à cirurgia descompressiva em um período superior a 72 horas, portanto novos trabalhos são necessários afim de comprovar a eficácia da cirurgia descompressiva nesses cães.

Dos 35 cães com DDIV toracolombar grau V, $8(22,8 \%)$ recuperaram-se totalmente, $7(17,2 \%)$ apresentaram recuperação parcial, em $10(28,5 \%)$ os sinais permaneceram inalterados, e $11(31,5 \%)$ foram submetidos à eutanásia pelos sinais neurológicos em um período de 6 meses por solicitação do proprietário. Destes 6 com recuperação parcialmente satisfatória, 2 desenvolveram andar espinhal (capacidade de sustentar o peso com os membros pélvicos,

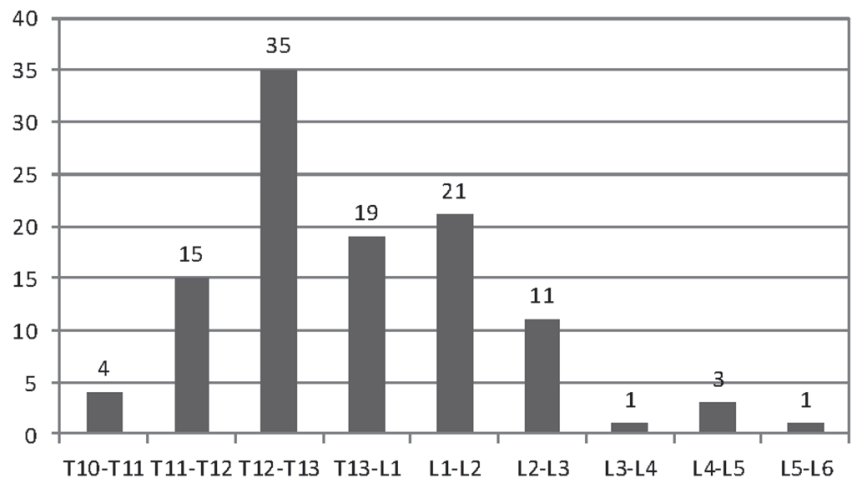

Fig.1. Distribuição de frequência dos discos intervertebrais afetados de 110 cães com doença do disco intervertebral toracolombar submetidos à cirurgia descompressiva.

$\begin{aligned} & \text { Quadro 1. Representação quanto ao grau de disfunção neurológica, } \\
& \text { recuperação funcional e recidiva dos sinais clínicos de } \mathbf{1 1 0} \text { cães com doença } \\
& \text { do disco intervertebral toracolombar submetidos à cirurgia descompressiva }\end{aligned}$
\begin{tabular}{lcccc}
\hline $\begin{array}{l}\text { Grau de disfunção } \\
\text { neurológica } \\
\text { (n=110) }\end{array}$ & $\begin{array}{c}\text { Satisfatória } \\
(\mathrm{n}=54)\end{array}$ & $\begin{array}{c}\text { Parcialmente } \\
\text { satisfatória (n=20) }\end{array}$ & $\begin{array}{c}\text { Insatisfatória } \\
(\mathrm{n}=36)\end{array}$ & $\begin{array}{c}\text { Recidiva } \\
(\mathrm{n}=9)\end{array}$ \\
\cline { 2 - 5 } Grau II (n=7) & $\mathrm{n}=5(71,4 \%)$ & $\mathrm{n}=1(14,3 \%)$ & $\mathrm{n}=1(14,3 \%)$ & $\mathrm{n}=3(42,8 \%)$ \\
Grau III (n=22) & $\mathrm{n}=15(68,2 \%)$ & $\mathrm{n}=4(18,2 \%)$ & $\mathrm{n}=3(13,6 \%)$ & $\mathrm{n}=2(9 \%)$ \\
Grau IV (n=46) & $\mathrm{n}=25(54,4 \%)$ & $\mathrm{n}=9(19,5 \%)$ & $\mathrm{n}=12(26,1 \%)^{\mathrm{a}}$ & $\mathrm{n}=2(4,3 \%)$ \\
Grau V (n=35) & $\mathrm{n}=8(22,8 \%)$ & $\mathrm{n}=6(17,2 \%)^{\mathrm{b}}$ & $\mathrm{n}=21(60 \%)^{\mathrm{c}}$ & $\mathrm{n}=2(5,7 \%)$
\end{tabular}

a Um cão foi submetido à eutanásia pelos sinais neurológicos após um mês da cirurgia por solicitação do proprietário, ${ }^{\mathrm{b}}$ Dois cães desenvolveram andar espinhal, ${ }^{\mathrm{c}} 11$ cães foram submetidos à eutanásia pelos sinais neurológicos em um período de seis meses por solicitação do proprietário. 
realizar movimentos de locomoção involuntários, no entanto, sem apresentar dor profunda), sendo considerados bons resultados da cirurgia pelos proprietários. Outros estudos demonstraram uma variação de $0-76 \%$ de recuperação considerada satisfatória em cães paraplégicos e sem dor profunda (Brisson 2010, Fingeroth \& Thomas 2015). Várias são as justificativas para explicar as diferenças nas taxas de recuperação (Brisson 2010), porém, neste estudo, a dificuldade em determinar e estimar a duração da perda da nocicepção contribuiu para o resultado. Kazakos et al. (2005) e Fingeroth \& Thomas (2015) questionaram o valor prognóstico da ausência da dor profunda em cães com DDIV toracolombar devido à interpretação subjetiva e verificaram que a velocidade da perda dos movimentos voluntários é mais precisa e o prognóstico é mais favorável para aqueles com perda progressiva da função motora. Devido a subjetividade da ausência de dor profunda no valor prognóstico, outros parâmetros estão sendo utilizados como a ressonância magnética (Ito et al. 2005), proteína Tau no líquido cerebroespinal (Roerig et al. 2013) e suas características (Levine et al. 2014, Chamisha et al. 2015).

Dos 110 cães, 74 (67,3\%) tiveram melhora dos sinais clínicos após o procedimento cirúrgico, sendo 54 (49,1\%) considerados satisfatórios e $20(18,2 \%)$, parcialmente satisfatórios. Destes cães, seis (8,1\%) estavam em grau II, 19 $(25,7 \%)$ em grau III, $35(47,3 \%)$ em grau IV e $14(18,9 \%)$ em grau V. Esses resultados são semelhantes aos descritos por Arias et al. (2007) e Festugatto et al. (2008), demonstrando que quanto menor o grau de disfunção neurológica com o qual o paciente é submetido à cirurgia descompressiva, melhor o seu prognóstico quanto à recuperação funcional. Se os cães em grau V fossem excluídos, o índice de resposta satisfatória para os demais graus de disfunção elevaria-se para $80 \%$ dos casos.

A precisão relatada com uso da mielografia é de 72-97\% para a localização da lesão e 53-100\% quando as extrusões discais são lateralizadas (Gibbons et al. 2006, Bos et al. 2007, Newcomb et al. 2012, Marinho et al. 2014). No presente estudo, as projeções oblíquas na mielografia forneceram informações relevantes, contribuindo com as outras projeções radiográficas habituais para localizar a compressão medular e foram imprescindíveis para definir o local de compressão medular, que seriam inconclusivas ou passadas despercebidas caso não fossem realizadas. Assim como no estudo de Zardo et al. (2010), em que as projeções oblíquas foram decisivas para encontrara lesão medular em $58,6 \%$ dos casos ( $\mathrm{n}=68)$, no presente estudo, as projeções oblíquas foram essenciais para localização correta em 40 casos (37\%). Segundo Gibbons et al. (2006), é necessária a realização das projeções oblíquas para a correta identificação da compressão medular, especialmente quando esta se apresenta lateralizada.

No estudo de Aikawa et al. (2012), 81 de 662 cães $(12,2 \%)$ apresentaram recidivas, sendo 15 confirmados durante a cirurgia, e 66 cães tiveram sinais clínicos de recidiva; entretanto, melhoraram com o tratamento clínico. Resultados semelhantes foram observados no presente estudo, $9(8,1 \%)$ cães apresentaram recindivas, sendo que 5 apresentaram melhora com o tratamento clínico e
4 foram submetidos ao tratamento cirúrgico pela segunda vez. Embora o baixo número de cães com recidiva, indica-se a fenestração do disco intervertebral afetado durante a descompressão medular toracolombar; pois permite que resquícios do núcleo pulposo do espaço intervertebral sejam retirados (Brisson 2010, Fingeroth \& Thomas 2015). A prevalência de recidivas em discos não fenestrados foi de 26 vezes maior que nos discos fenestrados (Aikawa et al. 2012). Embora complicações relacionadas à fenestração profilática, como introdução iatrogênica do material do disco intervertebral dentro do canal vertebral e subluxação e instabilidade vertebral, possam ocorrer, essa opção pode ser considerada um tratamento seguro e eficaz para prevenir recidivas em cães (Fingeroth \& Thomas 2015). Os 101 cães restantes não apresentaram recidiva dos sinais clínicos após a cirurgia até o presente momento (abril de 2015).

\section{CONCLUSÕES}

Diante dos resultados obtidos, pode-se concluir que:

- cadelas da raça Dachshund com média de seis anos de idade foram mais acometidas;

- o tratamento cirúrgico promove recuperação funcional satisfatória na maioria dos cães com DDIV toracolombar;

- o prognóstico para a recuperação funcional após o tratamento cirúrgico é melhor quanto menor o grau de disfunção neurológica;

- a percentagem de recidiva é baixa quando submetidos à terapia cirúrgica.

\section{REFERÊNCIAS}

Aikawa T., Fujita H., Shibata M. \& Takahashi T. 2012. Recurrent thoracolumbar intervertebral disc extrusion after hemilaminectomy and concomitant prophylactic fenestration in 662 chondrodystrophic dogs. Vet. Surg. 41(3):381-390.

Arias M.V.B., Nishioka C.M., Garcia C.O., Reia A.Z., Baraúna Júnior D. \& Marcasso R.A. 2007. Avaliação dos resultados clínicos após cirurgia descompressiva em cães com doença de disco intervertebral. Arq. Bras. Med. Vet. Zootec. 59(6):1445-1450.

Bos A.S., Brisson B.A., Holmberg D.L. \& Nykamp S.G. 2007. Use of the ventrodorsal myelographic view to predict lateralization of extruded disk material in small-breed dogs with thoracolumbar intervertebral disk extrusion: 104 cases (2004-2005). J. Am. Vet. Med. Assoc. 230(12):18601865.

Brisson B.A. 2010. Intervertebral disc disease in dogs. Vet. Clin. North Am., Small Anim. Pract. 40(5):829-858.

Chamisha Y., Aroch I., Kuzi S., Srugo I., Bdolah-Abram T., Chai O., Christopher M.M., Merbl Y., Rothwell K. \& Shamir M.H. 2015. The prognostic value of cerebrospinal fluid characteristics in dogs without deep pain perception due to thoracolumbar disc herniation. Res. Vet. Sci. 100:189196.

Festugatto R., Mazzanti A., Raiser A.G., Pelizzari C., Salbego F.Z., Beckmann D.V., Pereira D.T. \& Santos R.P. 2008. Recuperação funcional de cães com doença do disco intervertebral toracolombar submetidos ao tratamento cirúrgico. Ciência Rural 38(8):2232-2238.

Fingeroth J.M. \& Thomas W.B. 2015. Advances in Intervertebral Disc Disease in Dogs and Cats. Wiley-Blackwell, Iowa. 321p.

Gibbons S.E., Macias C., De Stefani A., Pinchbeck G.L. \& McKee W.M. 2006. The value of oblique versus ventrodorsal myelographic views for lesion lateralization in canine thoracolumbar disc disease. J. Small Anim. Pract. 47(11):658-662.

Ingram E.A., Kale D.C. \& Balfour R.J. 2013. Hemilaminectomy for thoracolumbar Hansen Type I intervertebral disk disease in ambulatory dogs 
with or without neurologic deficits: 39 cases (2008-2010). Vet. Surg. 42(8):924-931.

Ito D., Matsunaga S., Jeffery N.D., Sasaki N., Nishimura R., Mochizuki M., Kasahara M., Fujiwara R. \& Ogawa H. 2005. Prognostic value of magnetic resonance imaging in dogs with paraplegia caused by thoracolumbar intervertebral disk extrusion: 77 cases (2000-2003). J. Am. Vet. Med. Assoc. 227(9):1454-1460.

Itoh H., Hara Y., Yoshimi N., Harada Y., Nezu Y., Yogo T., Ochi H., Hasegawa D., Orima H. \& Tagawa M. 2008. A retrospective study of intervertebral disc herniation in dogs in Japan: 297 cases. J. Vet. Med. Sci. 70(7):701706.

Kazakos G., Polizopoulou Z.S., Patsikas M.N., Tsimopoulos G., Roubies N. \& Dessiris A. 2005. Duration and severity of clinical sings as prognostic indicators in 30 dogs with thoracolumbar disk disease after surgical decompression. J. Vet. Med. A, Physiol. Pathol. Clin. Med. 52(3):147-152.

Kerwin S.C., Levine J.M. \& Hicks D.G. 2012. Thoracolumbar spine, p.449475. In: Tobias K.M. \& Johnston S.A (Eds), Veterinary Surgery: small animal. Elsevier Saunders, Missouri.

Kranenburg H.J., Grinwis G.C., Bergknut N., Gahrmann N., Voorhout G., Hazewinkel H.A. \& Meij B.P. 2013. Intervertebral disc disease in dogs. 2. Comparison of clinical, magnetic resonance imaging, and histological findings in 74 surgically treated dogs. Vet. J. 195(2):164-171.

Levine G.J., Cook J.R., Kerwin S.C., Mankin J., Griffin J.F., Fosgate G.T. \& Levine J.M. 2014. Relationships between cerebrospinal fluid characteristics, injury severity, and functional outcome in dogs with and without intervertebral disk herniation. Vet. Clin. Pathol. 43(3):437-446.

Marinho P.V.T., Minto B.W., Zani C.C., Souza E.S., Dal Pietro N.H.P.S., Souza J.A.L., Canola J.C. \& De Nardi A.B. 2014. Mielografia lombar no diagnós- tico de extrusão do disco intervertebral toracolombar em cães: estudo prospectivo. Semina, Ciênc. Agrárias 35(4):1871-1880.

Newcomb B., Arble J., Rocha T.M., Pechman R. \& Payton M. 2012. Comparison of computed tomography and myelography to a reference standard of computed tomographic myelography for evaluation of dogs with intervertebral disc disease. Vet. Surg. 41(2):207-214.

Roerig A., Carlson R., Tipold A. \& Stein V.M. 2013. Cerebrospinal fluid tau protein as a biomarker for severity of spinal cord injury in dogs with intervertebral disc herniation. Vet J. 197(2):253-258.

Santos R.P., Beckmann D.V., Aiello G., Berté L., Ripplinger A., Polidoro D. \& Mazzanti A. 2011. Recuperação funcional em cães com doença do disco intervertebral toracolombar sem percepção à dor profunda: 37 casos (2002-2010). Pesq. Vet. Bras. 31(4):345-349.

Santos R.P., Beckmann D.V., Aiello G., Berté L., Ripplinger A., Polidoro D. \& Mazzanti A. 2012. Recuperação funcional de cães paraplégicos com doença do disco intervertebral toracolombar sem percepção à dor profunda submetidos ao tratamento cirúrgico: 15 casos (2006-2010). Pesq. Vet. Bras. 32(3):243-246.

Sharp N.J.H. \& Wheeler S.J. 2005. Small Animal Spinal Disorders. Elsevier Mosby, Edinburgh. 379p.

Smolders L.A., Bergknut N., Grinwis G.C., Hagman R., Lagerstedt A.S., Hazewinkel H.A., Tryfonidou MA. \& Meij B.P. 2013. Intervertebral disc degeneration in the dog. 2. Chondrodystrophic and non-chondrodystrophic breeds. Vet. J. 195(3):292-299.

Zardo K.M., Provasi A., Selmi A.L. \& Andrade Neto J.P. 2010. Contribuição das projeções oblíquas em mielografias de pequenos animais para a localização de lesões medulares causadas por processo degenerativo do disco intervertebral. Ciênc. Rural 40(11):2324-2331. 